
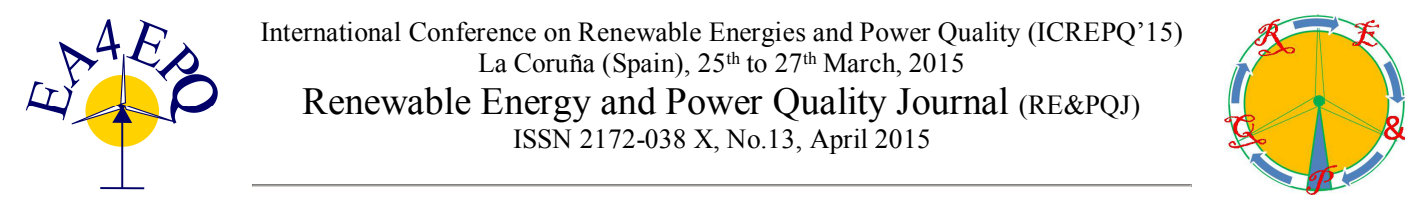

\title{
Observability of a 2-branch Double-Layer-Capacitor
}

\author{
F. Alonge ${ }^{1}$, M. Cirrincione ${ }^{2}$, G. Vitale ${ }^{3}$, G. Rodonò ${ }^{1}$ \\ ${ }^{1}$ Department of Energy, Information engineering and Mathematical models \\ D.E.I.M., University of Palermo, Viale delle Scienze - 90128 - Palermo (Italy) \\ Phone number +39-091-238.63.602, e-mail: francesco.alonge@unipa.it, giulio.rodono@unipa.it \\ 2 School of Engineering and Physics (SEP), The University of the South Pacific \\ Laucala Campus, Suva, Fiji Islands, e-mail: cirrincione_m@usp.ac.fj \\ ${ }^{3}$ Institute of Intelligent Systems for Automation (ISSIA), National Research Council of Italy. \\ Via Dante Alighieri n. 12 - 90141 - Palermo (Italy), e-mail: vitale@pa.issia.cnr.it
}

\begin{abstract}
The paper proposes a study of the observability of a 2-branch Double-Layer Capacitor (DLC) mathematical model, derived from its well known 2-branch nonlinear physical model. The study is a basis to perform the diagnosis of the system by employing observers. The analysis is carried out by means of the sufficient rank condition of the observability matrix for nonlinear models. It is shown that the rank condition is satisfied everywhere in the two-dimensional state space, except for a characteristic curve in the plane defined by the two state variables. This curve is usually outside the usual range of operation of Double-Layer Capacitors available on the market. Finally, it is shown that the above curve is sensitive to the resistance of the branch containing the nonlinear capacitance, i.e. the faster branch. In particular, increasing this resistance can cause the curve to intersect the region of usual range of operation of a DLC.
\end{abstract}

Keywords. DLC, SC, supercapacitor, observability, state-space, diagnosis.

\section{List of variables}

$\quad$ Variables(s)
$I_{d}$
$C_{1, a}$
$C_{2, a}$
$k_{1}$
$x_{1}=V_{1}=V_{1}(t)$
$x_{2}=V_{2}=V_{2}(t)$
$\dot{V}_{1}=d V_{1} / d t$
$\dot{V}_{2}=d V_{2} / d t$
$C_{1, b}=k_{1} V_{1}$
$C_{1}=C_{1, a}+C_{1, b}$
$Q_{1}=C_{1} x_{1}$
$Q_{2}=C_{2} x_{2}$
$i_{1}=i_{1}(t)$

$$
\begin{aligned}
& i_{2}=i_{2}(t) \\
& i_{3}=i_{3}(t) \\
& u \\
& y \\
& f(x), g(x), h(x) \\
& O(x)
\end{aligned}
$$

Current (branch-2)

Current (branch-3) A

Input of the system

Output

Smooth functions

Observability Matrix

\section{Introduction}

Supercapacitors (SC) are widely recognized as fast and efficient energy storage solutions, especially in power applications thanks to their power densities (typically larger than the ones reachable with batteries) and their energy densities (10 to 20 times larger than in electrolytic capacitors). Among them, the so-called Double-LayerCapacitor (DLC) is available on the market with rated voltage of about $2.3 \mathrm{~V}$ and capacitance values up to $1500 \mathrm{~F}$; anyway, higher voltages can be obtained by suitable seriesparallel combinations of such single DLC units [1].

DLCs use polymer foils, which are able to offer a great density of electric charges; unfortunately, this characteristic implies an equivalent model more complicated than the classic capacitor model. In the literature, models based on electrochemical laws [2]-[3], equivalent electrical circuits [4]-[5], and model based on impedance measurement [6][7], are proposed.

Among these models, the simplified physical model proposed in [4] it is sufficient for practical engineering applications, over the range of some tens of minutes [1].

However, due to the high energy stored during operation, a diagnostic system is required for the system reliability, able to recognize the behavior of the SC in terms of the stored charge, based on electrical quantities measured at the SC terminals. As well known, in order to achieve this 
objective, the observability of the SC model must be studied, which is not an easy task, since the SC model is nonlinear.

This paper, aims at the observability of a DLC, whose physical model consists of two parallel-connected branches: the former consists of a parallel connection of a voltage dependent capacitor and a conventional one, in a series with a resistor, the latter branch consists of a series connection of a linear resistor and a linear capacitor.

More precisely, a package consisting of several SC cells parallel and series connected between them is considered, with the aim of obtaining the desired values of current and voltages required in the practical applications. However, assuming that all the cells are identical, the physical scheme of the whole package coincides with that of a single cell. It is also assumed that the parameters of this cell have been already identified using, for example, the approach described in [12].

The study is based on the sufficient rank condition of the observability matrix for nonlinear models. In the following, it is demonstrated that the rank condition is satisfied in the whole two-dimensional state space, except for a nonobservability curve in the plane defined by the two state variables. By doing this, it is possible to highlight that the observability is satisfied for the considered DLCs available on the market. Finally, it is shown that a strong increase of internal resistance can cause the non-observability curve to enter inside the region of the usual range of operation.

\section{Description of the Adopted Model}

In this section, the fundamentals of a DLC operation are presented; on this basis, the physical model is discussed and, finally, the state-space model is obtained.

\section{A. Model presentation}

A DLC is quite different from a conventional electrolytic capacitor: indeed, it exhibits virtual plates that are actually two layers of the same substrate (see figure 1), realizing the phenomenon of the double-layer-effect. Their electrochemical properties, the so-called Helmholtz [9] "electrical double layer", result in the effective separation of charges despite the infinitely thin (about nanometres) physical separation of the layers. The material widest used is the "activated carbon" [10].

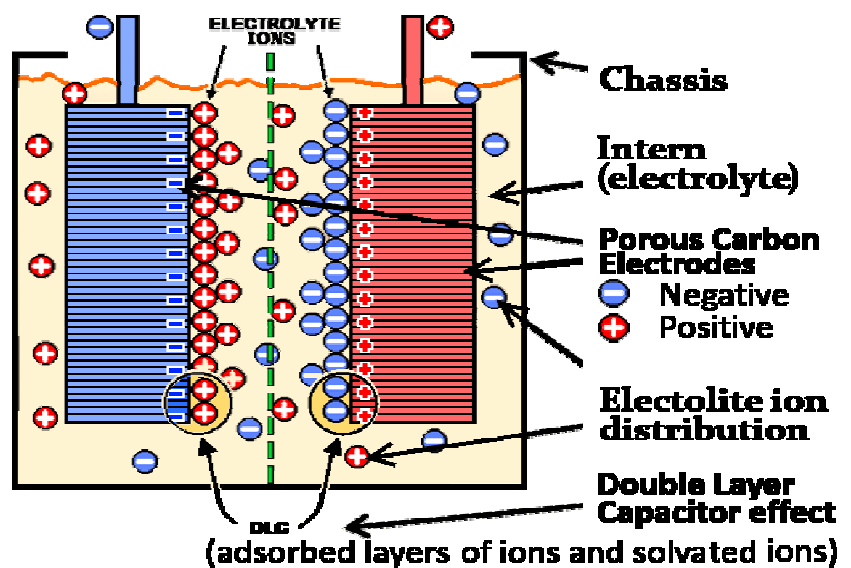

Fig. 1. A Double-Layer-Capacitor scheme.
The absence of need for a great layer of dielectric, and the porosity of the used material, permit plates to be packed with much larger surface area in a given volume, resulting in high capacitances and in practical-sized packages. In an electrical double layer, each layer by itself is quite conductive, but at the interface, where the layers are effectively in contact, no significant current flows between the layers.

The double layer can tolerate only low voltages, which means that electric double-layer capacitors, if rated for higher voltages, must be made up of series-connected DLCs, like series-connected cells in higher-voltage batteries. In general, DLCs have much higher power density [8] than batteries.

\section{B. Model finding}

A multi RC-branch circuit is made up of a certain number of RC-series branches; in DLC case, recalling that $\tau_{i}=R_{i} C_{i}$ [11], every branch simulates a separated behavior during time.

The more common models adopted in literature are the 2branch [4]-[12] and the 3-branch [13]-[1] ones; the first, being simpler, leads up to less complex computations; the second, although more complicated, approximate the behaviour of a real DLC better. However, for power applications, a simpler 2 branches model with a voltage dependent capacitor series connected with a resistor and a series of a linear resistor with a linear capacitor is adopted [1]-[6]. For this reason, in this paper, only the 2-branch model is studied.

Figure 2 shows a schema of the DLC equivalent circuit. It can be noticed that the first branch contains the voltage dependent capacitor; in particular, it is modeled by a constant term, parallel connected to a linear voltage dependent capacitor. This implies a nonlinearity that justifies the observability study. In addition, the voltage at the capacitor terminals is a state variable, but it does not coincide with the voltage at the external terminals due to the presence of the series resistance.

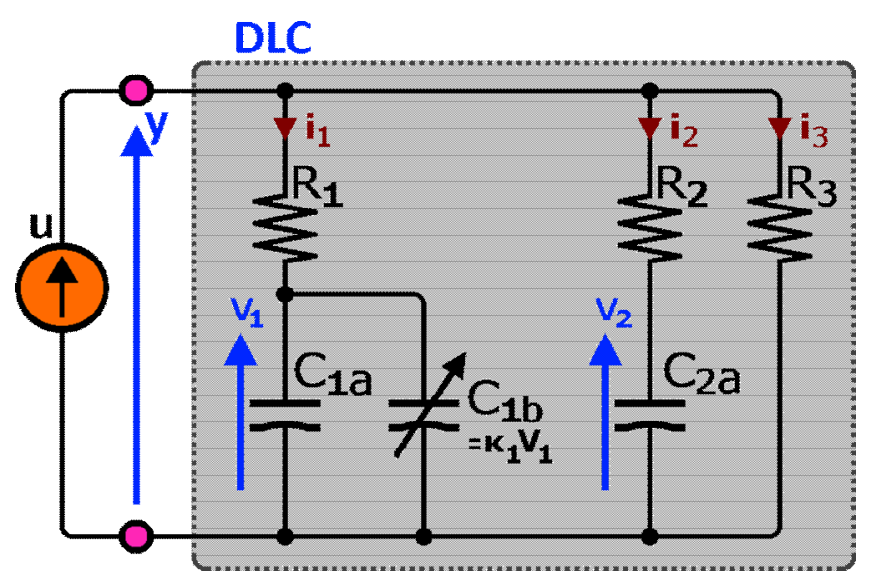

Fig. 2. Schematics of a DLC equivalent circuit. $u=I_{d}$ is the input current; $i_{1}, i_{2}, i_{3}$ are the currents; $R_{1}, R_{2}, R_{3}$ are the resistances; $C_{1, a}, C_{2, a}$ are the linear capacitances; $C_{1, \mathrm{~b}}=k_{1} V_{1}$ is the nonlinear capacitance; $R_{1}, R_{2}, R_{3}, C_{1, a}, C_{1, \mathrm{~b}}, C_{2, a}$ make the DLC; $y \equiv y_{1} \equiv y_{2} \equiv y_{3}$ is the output voltage. 


\section{Mathematical model of a DLC cell}

It is important to bear in mind that it must always be:

$$
V_{1} \geq 0 \quad, \quad V_{2} \geq 0
$$

This condition is imposed by the structure of the SC for which the applied voltage must always be positive. The upper limit of the state variables is given by the rated voltage of the SC. Now, by a simple analysis, it is possible to find the mathematical equivalent model starting from the following equations:

$$
\begin{array}{ll}
C_{1}=C_{1, a}+k_{1} V_{1}(t) & \text { branch-1 } \\
C_{2}=C_{2, a} & \text { branch-2 } \\
Q_{1}=C_{1} V_{1}=C_{1, a} V_{1}+k_{1} V_{1}^{2} & \text { branch-1 } \\
Q_{2}=C_{2} V_{2}=C_{2, a} V_{2} & \text { branch-2 } \\
\frac{d Q_{1}}{d V_{1}}=C_{1, a}+2 k_{1} V_{1}=C_{1, d} & \text { branch-1 } \\
\frac{d Q_{2}}{d V_{2}}=C_{2, a} & \text { branch-2 } \\
i_{1}=\frac{d Q_{1}}{d V_{1}} \frac{d V_{1}}{d t}=\ldots=C_{1, d} \dot{V}_{1} & \text { branch-1 } \\
i_{2}=\frac{d Q_{2}}{d V_{2}} \frac{d V_{2}}{d t}=\ldots=C_{2, \mathrm{a}} \dot{V}_{2} & \text { branch-2 }
\end{array}
$$

where $C_{1, d}$ is the differential capacitance of first branch.

Now, placing $x=\left[\begin{array}{ll}x_{1} & x_{2}\end{array}\right]^{T}=\left[\begin{array}{ll}V_{1} & V_{2}\end{array}\right]^{T}$, it is easy to verify that the mathematical model results as:

$$
\left\{\begin{array}{l}
\dot{x}=f(x)+g(x) u \\
y=h(x)
\end{array}\right.
$$

with:

$$
\begin{gathered}
f(x)=\left[\begin{array}{cc}
-\frac{R_{2}+R_{3}}{\gamma} \frac{1}{C_{1, d}} & \frac{R_{3}}{\gamma} \frac{1}{C_{1, d}} \\
\frac{R_{3}}{\gamma C_{2, \mathrm{a}}} & \frac{R_{1}+R_{3}}{\gamma C_{2, \mathrm{a}}}
\end{array}\right]\left[\begin{array}{l}
x_{1} \\
x_{2}
\end{array}\right] \\
g(x)=\left[\begin{array}{cc}
\frac{R_{2} R_{3}}{\gamma} \frac{1}{C_{1, d}} \\
\frac{R_{1} R_{3}}{\gamma C_{2, \mathrm{a}}}
\end{array}\right] \\
h(x)=\left[\begin{array}{ll}
\frac{R_{2} R_{3}}{\gamma} & \left.\frac{R_{1} R_{2}}{\gamma}\right]\left[\begin{array}{l}
x_{1} \\
x_{2}
\end{array}\right]+\frac{R_{1} R_{2} R_{3}}{\gamma} u
\end{array}\right.
\end{gathered}
$$

and:

$$
\gamma=R_{2} R_{3}+R_{1} R_{3}+R_{1} R_{2}
$$

\section{Observability}

In this section, the observability matrix is calculated; therefore, imposing the observability condition, the operating region - in which the system is observable - is identified.

As already said, the study the observability is useful for diagnostic purposes, since it permits to deduce the charge levels $Q_{i}$, and then the status of operations of SC, by means of the knowledge of the states $x_{i}$.

In particular, from the voltage on the two capacitors (see figure 2) it is possible to calculate the stored charge. During the normal operation, the voltage at the capacitor terminals must be coherent with the capacitance and the flowing current. If deterioration occurs, the capacitance value and, consequently, the stored charge will diminish.

In order to study the observability of the model system, it is necessary to determine the observability matrix and verify the rank condition; if it is satisfied the model is observable, contrarily the observability is not guaranteed [14]-[15]. The observability matrix is given by:

$$
\begin{aligned}
& O(x)=\left[\begin{array}{c}
d h \\
d L_{f} h
\end{array}\right]= \\
& =\left[\begin{array}{cc}
\alpha_{1} & \alpha_{2} \\
\frac{\alpha_{3} x_{1}^{2}+\alpha_{4} x_{1}+\alpha_{5} x_{2}+\alpha_{6}}{\left(\alpha_{7} x_{1}+\alpha_{8}\right)^{2}} & \frac{\alpha_{9} x_{1}+\alpha_{10}}{\alpha_{7} x_{1}+\alpha_{8}}
\end{array}\right]
\end{aligned}
$$

with:

$$
\begin{aligned}
& \alpha_{1}=\mathrm{R}_{2} \mathrm{R}_{3} / \gamma \\
& \alpha_{2}=\mathrm{R}_{1} \mathrm{R}_{3} / \gamma \\
& \alpha_{3}=4 R_{1} R_{3}^{2} k_{1}^{2} \\
& \alpha_{4}=4 R_{1} R_{3}^{2} k_{1} C_{1, a}=\alpha_{3} C_{1, a} / k_{1} \\
& \alpha_{5}=-2 R_{1} R_{3}^{2} k_{1} C_{2, a}=-\alpha_{3} C_{2, a} /\left(2 k_{1}\right) \\
& \alpha_{6}=-R_{3} C_{1, a}\left(R_{2}^{2} C_{2, a}-R_{1} R_{3} C_{1, a}+R_{2} R_{3} C_{2, a}\right) \\
& \alpha_{7}=2 k_{1} C_{2, a} \gamma^{2} \\
& \alpha_{8}=C_{1, a} C_{2, a} \gamma^{2} \\
& \alpha_{9}=-2 R_{1} R_{3} k_{1}\left(R_{1}+R_{3}\right) \\
& \alpha_{10}=C_{1, a} C_{2, a} \gamma^{2}
\end{aligned}
$$

According to [16], the model (6) is observable if the determinant of matrix $O(x)$ is different from zero for all $x$. This determinant is given by:

$$
\begin{aligned}
& \operatorname{det}(O(x))= \\
& =\frac{\alpha_{11} x_{1}^{2}+\alpha_{12} x_{1}+\alpha_{13} x_{2}+\alpha_{14}}{\gamma\left(\alpha_{5} x_{1}+\alpha_{6}\right)^{2}}
\end{aligned}
$$

with:

$$
\alpha_{11}=[\ldots]=-4 R_{1} R_{3}^{2} \gamma k_{1}^{2}
$$




$$
\begin{aligned}
\alpha_{12} & =[\ldots]=-2 R_{3}^{2} k_{1}\left(2 R_{1}^{2} C_{1, a}\left(R_{2}+R_{3}-R_{2} R_{3}^{2} C_{2, a}\right)\right) \\
\alpha_{13} & =[\ldots]=2 R_{1} R_{2} R_{3} k_{1} C_{2, a} \\
\alpha_{14} & =[\ldots]=-R_{3}^{2} \gamma C_{1, a}\left(R_{1} C_{1, a}-R_{2} C_{2, a}\right)
\end{aligned}
$$

Eq. 12 is different from zero for all $x$ such that:

$$
x_{2} \neq a x_{1}^{2}+b x_{1}+c
$$

where $a, b$, and $c$ are:

$$
\begin{aligned}
& a=2 \gamma k_{1} /\left(R_{2} C_{2, a}\right) \\
& b=\left(2 R_{1} \gamma C_{1, a}-R_{2}^{2} R_{3} C_{2, a}\right) /\left(R_{1} R_{2} R_{3} C_{2, a}\right) \\
& c=R_{1} \gamma\left(R_{1} C_{1, a}-R_{2} C_{2, a}\right) /\left(R_{2} C_{2, a}\right)
\end{aligned}
$$

The states in which the rank condition is not satisfied belong to a non-observability parabola, defined as:

$$
x_{2}=a x_{1}^{2}+b x_{1}+c
$$

On this curve the observability is not assured since (14) represents only a sufficient condition. Therefore, for any SC described by the proposed model, the observability can be studied by drawing the parabola described by eq. (14), on the plane defined by the state variables and verifying that the trajectory of the operation point does not cross the curve defined by (14).

It should be noted that the coefficient $a$ is always greater than zero, which implies that the parabola concavity is upwards.

\section{A. Operating Conditions}

The operating region of a SC is defined by its rated voltage. In particular, it is a rectangle on the plane defined by state variables whose boundaries are equal to the rated voltage of the SC. The parabola (14) is identified by its characteristic points as the intersection with the axes and the vertex. It should not be inside the DLC working region.

Hence, best conditions for the observability are: a parabola completely on the right side of the operating region or, alternatively, a parabola inside which the DLC working region lies.

With reference to figure 3 , some characteristics points are defined. They are the intersection points with axis $\mathrm{x}_{1}=0 \mathrm{P}_{1}$ and $\mathrm{P}_{2}$, the intersection with axis $\mathrm{x}_{2}=0 \mathrm{P}_{3}$ and its vertex $\mathrm{P}_{\mathrm{V}}$.

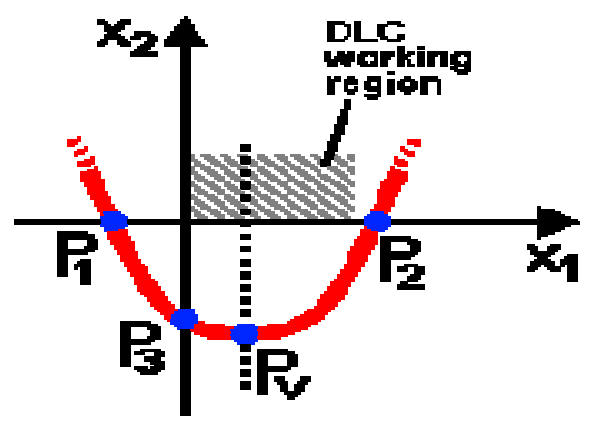

Fig. 3. A generic parabola and its characteristic intersection points: $P_{1}$ and $P_{2}$ with axis $x_{1}=0, P_{3}$ with axis $x_{2}=0$, and its vertex $P_{V}$.
Placing that $V_{N}=V_{1, N}=V_{2, N}$, some simple calculations allow, in the scond case, the contraint to be identified as:

$$
V_{N}<\frac{\left(b^{2}-4 a c\right)^{2} b}{2 a}
$$

with the discriminant:

$$
\Delta=a^{2}-4 a c
$$

\section{DLC Examples}

On this section, some numerical examples are presented. The characteristic parabola is calculated for some SCs available on the market. Note: values are approximated, $R_{3}$ is expressed in $k \Omega$, "E." means $\operatorname{Epcos}^{\mathrm{TM}}$ [4], "M." means Maxwell ${ }^{\mathrm{TM}}$ [4], [12].

Table I. DLC parameters and values sets for the Epcos ones and for the Maxwell one.

\begin{tabular}{|l|r|r|l|l|l|c|}
\cline { 2 - 7 } \multicolumn{1}{c|}{} & $R_{1}$ & $R_{2}$ & $R_{3}$ & $C_{1, a}$ & $C_{2, a}$ & $k_{1}$ \\
\hline E. 110 F (a) & 0,01 & 17,5 & 5 & 89 & 14 & 29,1 \\
\hline E. 110 F (b) & 0,01 & 18,3 & 5 & 84,7 & 13 & 27,4 \\
\hline E. 200 F (a) & 0,009 & 8,8 & 5 & 158 & 27,6 & 56,2 \\
\hline E. 200 F (b) & 0,009 & 7,8 & 5 & 152,7 & 30,8 & 58,7 \\
\hline M. 350 F (a) & 0,005 & 5,5 & 2,5 & 232,5 & 43,2 & 89,9 \\
\hline M. 350 F (b) & 0,004 & 7,8 & 2,5 & 234,7 & 30,6 & 82,2 \\
\hline E. 600 F & 0,003 & 3,1 & 2,5 & 454,5 & 77,4 & 176 \\
\hline M. 83 F & 0,01 & 11 & 1,1 & 39,73 & 11,8 & 0,9 \\
\hline
\end{tabular}

Substituting values of Table I in (14), Table II and Table III are found (approximate values); Table II shows values of a b c for all DLCs, Table III shows the equations and the $P_{i}$ and $P_{V}$ points. The values are also plotted in figure 4 , figure 5, figure 6 , in which all $P_{i}$ and all $P_{V}$ satisfy constraints given by eqs.15-18. Furthermore, each parabola does not touch real operating regions. Thus, it can be claimed that all studied DLCs 2-branch models are observable.

All parabolas are shown in figure 4; it can be noted that the first seven DLCs are quite similar to each other, and significantly different from the last one.

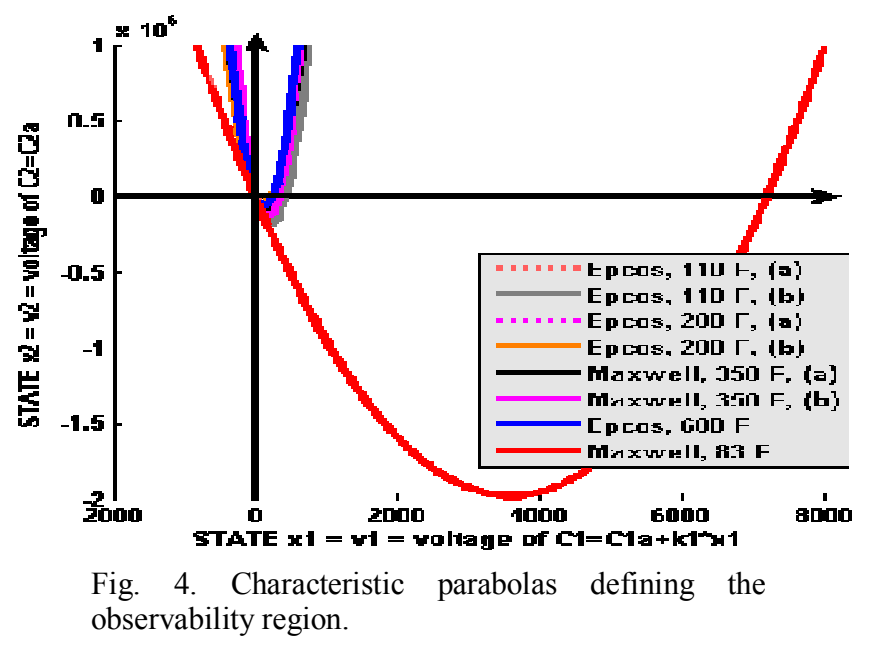


Table II. Coefficients $a, b$ and $c$ of each DLCs.

\begin{tabular}{|l|c|c|c|}
\cline { 2 - 4 } \multicolumn{1}{c|}{} & $a$ & $b$ & $c$ \\
\hline E. 110 F (a) & 4.25 & -1728 & -2655 \\
\hline E. 110 F (b) & 4.18 & -1737 & -2699 \\
\hline E. 200 F (a) & 4.08 & -978.9 & -1385 \\
\hline E. 200 F (b) & 3.82 & -850.7 & -1115 \\
\hline M. 350 F (a) & 4.16 & -1146 & -1491 \\
\hline M. 350 F (b) & 5.37 & -1740 & -2497 \\
\hline E. 600 F & 4.56 & -1076 & -1395 \\
\hline M. 83 F & 0.15 & -1090 & -24161 \\
\hline
\end{tabular}

Table III. Parabola equations and its characteristic points obtained for each DLC.

\begin{tabular}{|c|c|c|c|c|}
\hline & \multicolumn{4}{|c|}{$\begin{array}{lll}\text { Equations } / & P_{1}, P_{2}, P_{3}, P_{V}\end{array}$} \\
\hline & $P_{1}$ & $P_{2}$ & $P_{3} \cdot 10^{3}$ & $P_{V} \cdot 10^{3}$ \\
\hline \multirow{2}{*}{$\begin{array}{c}\text { E. } 110 \\
\text { F (a) }\end{array}$} & \multicolumn{4}{|c|}{$x_{2}=4.246507 x_{1}^{2}-1728.447 x_{1}-2654.953$} \\
\hline & $(-1.5 ; 0)$ & $(408.6 ; 0)$ & $(0 ;-2.6)$ & $(0.2 ;-176)$ \\
\hline \multirow{2}{*}{$\begin{array}{c}\text { E. } 110 \\
\text { F (b) }\end{array}$} & \multicolumn{4}{|c|}{$x_{2}=4.183435 x_{1}^{2}-1737.109 x_{1}-2699.373$} \\
\hline & $(-1.55 ; 0)$ & $(408.6 ; 0)$ & $(0 ;-2.7)$ & $(0.2 ;-180)$ \\
\hline \multirow{2}{*}{$\begin{array}{c}\text { E. } 200 \\
\text { F (a) }\end{array}$} & \multicolumn{4}{|c|}{$x_{2}=4.082322 x_{1}^{2}-978.9151 x_{1}-1384.849$} \\
\hline & $(-1.4 ; 0)$ & $(241.2 ; 0)$ & $(0 ;-1.4)$ & $(0.19 ;-59)$ \\
\hline \multirow{2}{*}{$\begin{array}{c}\text { E. } 200 \\
\text { F (b) }\end{array}$} & \multicolumn{4}{|c|}{$x_{2}=3.81571 x_{1}^{2}-850.7151 x_{1}-1114.561$} \\
\hline & $(-1.3 ; 0)$ & $(224.1 ; 0)$ & $(0 ;-1.1)$ & $(0.11 ;-47)$ \\
\hline \multirow{2}{*}{$\begin{array}{c}\text { M.350 } \\
\text { F (a) }\end{array}$} & \multicolumn{4}{|c|}{$x_{2}=4.164707 x_{1}^{2}-1146.061 x_{1}-1490.954$} \\
\hline & $(-1.3 ; 0)$ & $(276.5 ; 0)$ & $(0 ;-1.5)$ & $(0.14 ;-79)$ \\
\hline \multirow{2}{*}{$\begin{array}{c}\text { M.350 } \\
\text { F (b) }\end{array}$} & \multicolumn{4}{|c|}{$x_{2}=5.369844 x_{1}^{2}-1740.077 x_{1}-2497.262$} \\
\hline & $(-1.43 ; 0)$ & $(325.5 ; 0)$ & $(0 ;-2.5)$ & $(0.16 ;-140)$ \\
\hline \multirow{2}{*}{$\begin{array}{c}\text { E. } 600 \\
\text { F }\end{array}$} & \multicolumn{4}{|c|}{$x_{2}=4.562295 x_{1}^{2}-1076.478 x_{1}-1394.961$} \\
\hline & $(-1.3 ; 0)$ & $(237.2 ; 0)$ & $(0 ;-1.4)$ & $(0.12 ;-63)$ \\
\hline \multirow{2}{*}{$\begin{array}{c}\text { M.83 } \\
\text { F }\end{array}$} & \multicolumn{4}{|c|}{$x_{2}=0.1520386 x_{1}^{2}-1090.288 x_{1}-24161.45$} \\
\hline & $(-22.1 ; 0)$ & $193.2 ; 0)$ & $(0 ;-2.4)$ & $(3.58 ;-1914)$ \\
\hline
\end{tabular}

A zoom of the parabolas near $P_{2}$ points is shown in figure. 5 ; it is important to notice that all parabolas pass through the first quadrant for values of $x_{1}$ greater than $241.2 \mathrm{~V}$, which is really greater than real possible values of $x_{1}$ (just some volts).

Figure 6 shows a zoom in the neighbourhoods of the origin of the state plane. It can be seen that the parabola never crosses the working region in the first quadrant, but touches just $2^{\text {nd }}, 3^{\text {rd }}$ and $4^{\text {th }}$ quadrant.

Finally, it can be argued that the studied SCs, within the operating values of the states, are always observable.

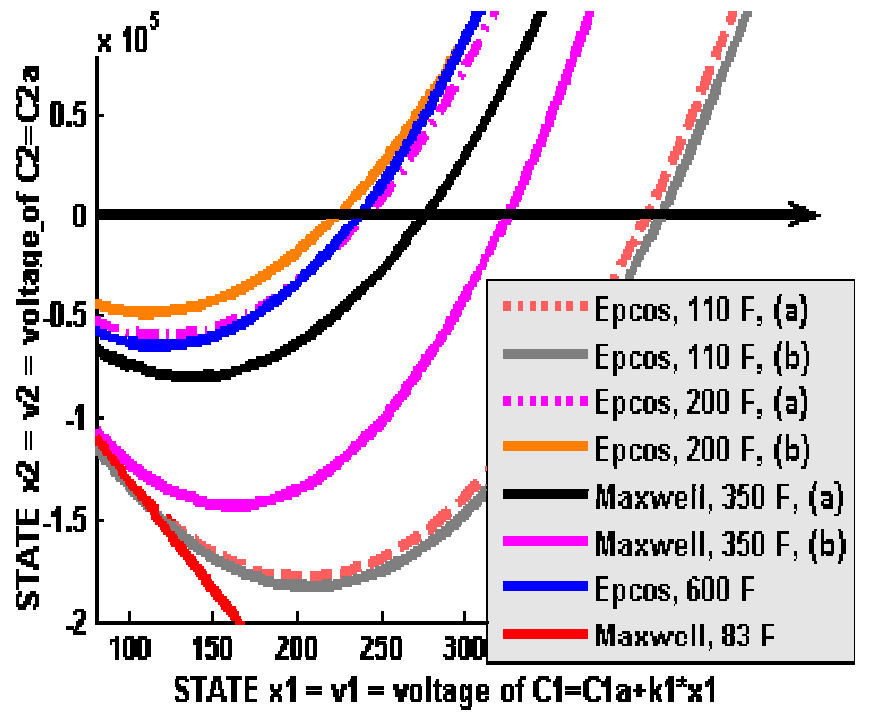

Fig. 5. Each right arm of all parabolas showing that they are all outside the operating region.

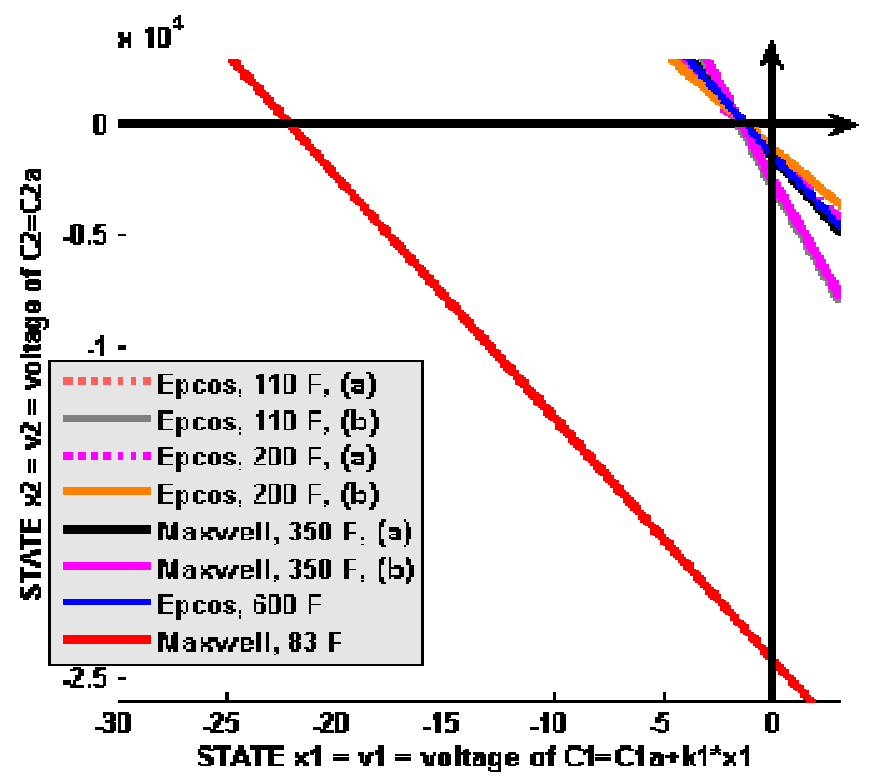

Fig. 6. Left arm of all parabolas showing that they do not cross the first quadrant.

\section{Parametric Analysis}

The observability analysis proposed in section IV has shown that during the normal operating conditions, all the considered SCs are observables.

By observing the model of figure 2, it is possible to note that the voltage at the SC terminals differs from the voltage at the nonlinear capacitance for the presence of the series resistance $R_{l}$. As in [12], the value of $R_{l}$ is given by manufacturer; if necessary, a possible method of computation is the amperometric measurement. Since this parameter is the cause by while the terminal voltage is different from the voltage at the terminal of the capacitance $C_{l}$, it is useful to investigate if an increase of $R_{l}$ can affect significantly the observability. To this aim, a fictitious increase of $R_{l}$ has been introduced. In particular, with reference to SC Epcos mod. A, it has been increased up to 
reach the working region. It occurs when $R_{l}=0.55 \Omega$. It corresponds to the condition: $P_{2, \min }=V_{1, N} \simeq 2.3 \mathrm{~V}$.

Results are collected into table III and drawn in figure 7. Only $1^{\text {st }}$ quadrant is considered, because of it contains DLC operating region (other quadrants are drawn in grey color). Overcoming $R_{1}$, parabolas go through DLC operating region (black diagonal lines) and its are drawn in red color; here, nothing can be said about observability.

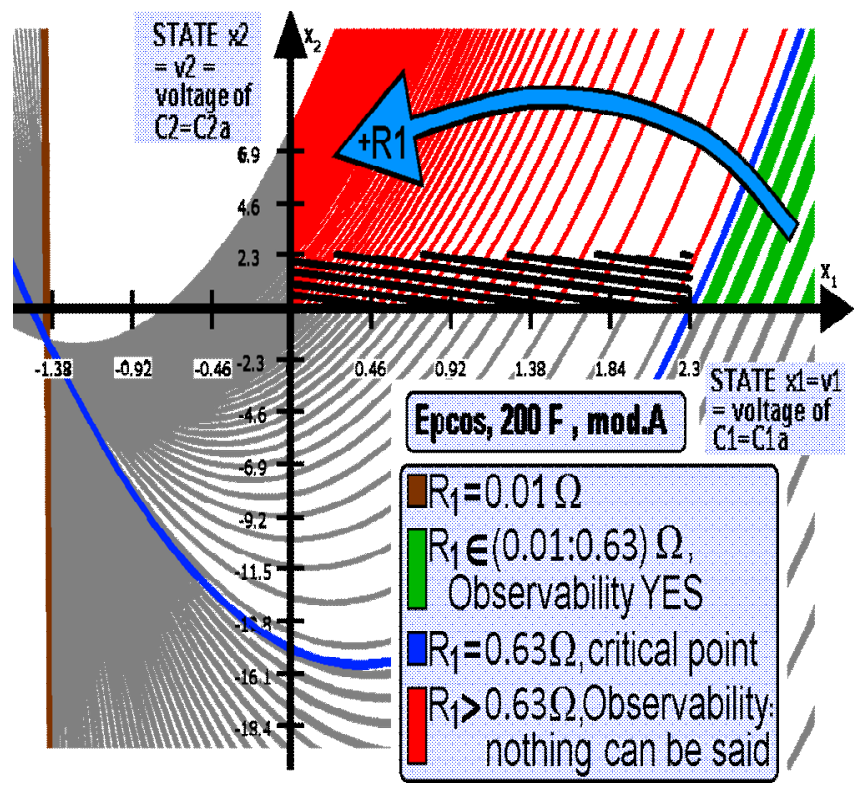

Fig. 7. Observability for green regions, nothing can be said for observability inside red region. Black diagonal lines are DLC's operating region; gray curves are not of interest; green curves are of secure observability; for red curves, for x1 (0:241.2), nothing can be said.

From the above presented analysis, it can be deduced that, during operating conditions, the $\mathrm{SC}$ is observable. If degradation leads to an increase of $R_{l}$, it can be admitted until the characteristic parabola does not reach the operating region. A further increase of $R_{l}$ could imply that the locus of operating point crosses the characteristic parabola, in this case the observability is not guaranteed.

The internal resistance variations, during normal operation, do not affect the observability. As a matter of fact, it has been demonstrated that the internal resistance decreases while the DLC temperature increases; moreover, high operating frequencies cause a lessening of the same system [16] [17].

For example, for the Maxwell BCAP350 F DLC, the equation (20) has been found:

$$
R_{T}=R_{20} \frac{1+e^{-k_{T}\left(T-T_{20}\right)}}{2}
$$

where: $R_{T}$ is the resistance depending on temperature, $R_{20}$ is the resistance at $20^{\circ} \mathrm{C}, T$ is the surrounding temperature, $k_{T}$ the temperature coefficient $\left(k_{T}=0,0025^{\circ} \mathrm{C}\right)$.

For a temperature raise from $20^{\circ} \mathrm{C}$ to about $200^{\circ} \mathrm{C}$ (see figure 7), the increase of $\mathrm{R}_{\mathrm{T}}$ exceeds the critical value $(0,55 \Omega)$ for which the non-observability parabola intersects the DLC operating region.
On the other hand, an anomalous increase of the temperature reduces the life of the cell, because of electrolyte degradation and of separator instability. This critical condition can be monitored by the internal resistance estimation.

Table IV suggests a general procedure before performing the diagnostic. For a given SC, the non-observability parabola must be calculated. Then, if it does not cross the operating region, the upper limit of $R_{l}$ corresponding to a critical condition in which the observability cannot be guaranteed must be calculated.

Table IV. Parametric analysis of "Epcos-200F-(a)"; only green region grants observability.

\begin{tabular}{|c|c|c|}
\hline $\mathrm{R}_{1} /$ & Equation & $, \mathrm{P}_{2}, \mathrm{P}_{3}, \mathrm{P}_{\mathrm{V}}$ \\
\hline \multicolumn{3}{|c|}{$R_{1}=0.01 \cdot 1=0.01 \Omega$} \\
\hline \multicolumn{2}{|c|}{$x_{2} \simeq 4 x_{1}^{2}-979 x_{1}-1384.85$} & $P_{1}(-1.5 ; 0)$ \\
\hline$P_{2}(241.2 ; 0)$ & $P_{2}(0 ;-1384.8)$ & $P_{V}(119.9 ;-58 k)$ \\
\hline \multicolumn{3}{|c|}{$R_{1}=0.01 \cdot 63=0.63 \quad \Omega \quad$ critical } \\
\hline \multicolumn{2}{|c|}{$x_{2}=4.34 x_{1}^{2}-3.5 x_{1}-14.9$} & $P_{1}(-1.5 ; 0)$ \\
\hline$P_{2}(2.3 ; 0)$ & $P_{2}(0 ;-14.9)$ & $P_{V}(0.4 ; 0.12)$ \\
\hline \multicolumn{3}{|c|}{$R_{1}=0.01 \cdot 225=2.25 \Omega$} \\
\hline \multicolumn{2}{|c|}{$x_{2}=6.14 x_{1}^{2}+15.3 x_{1}+7.93$} & $P_{1}(-1.75 ; 0)$ \\
\hline$P_{2}(-0.74 ; 0)$ & $P_{2}(0 ; 7.93)$ & $P_{V}(-1.24 ;-9.9)$ \\
\hline
\end{tabular}

During normal operation, the internal resistance could be estimated by an observer; particular care has to be taken when the value of $R_{l}$ overcomes its critical value.

\section{Conclusions}

In this paper a study of the observability of a 2-branch Double-Layer Capacitor (DLC) model is proposed. The study is a basis to perform the diagnosis of the system. A nonlinear DLC model, suitable for power applications, is used. A mathematical model - placed in canonical form - is developed.

The proposed method can be applied to all SC whose model is composed of two parallel-connected branches in which the former is formed by a voltage dependent capacitor series connected with a resistor and the latter is a series of a linear resistor with a linear capacitor.

The analysis on some commercial SCs has demonstrated that all the 2-branch DLCs studied, are observable. On the other hand, a degradation leading to an increase of the series resistance of the nonlinear capacitor can jeopardize the observability of the system.

The method requires, for efficient operation, an estimation of the internal resistance that could be performed by a dedicated real-time observer.

\section{Appendix A}

For the model (6), assuming that $f(x): \mathbb{R}^{n} \rightarrow \mathbb{R}^{n}$, $g(x): \mathbb{R}^{n} \rightarrow \mathbb{R}^{n}$ and $h(x): \mathbb{R}^{n} \rightarrow \mathbb{R}$ are smooth vector 
fields, the observability matrix of the nonlinear model is given by:

$$
O(x)=\left[\begin{array}{c}
d h \\
d L_{f} h
\end{array}\right],
$$

where $d h$ is the gradient of $h$, and $d L_{f} h$ is the Lie derivative of $h$ along $f$, defined as:

$$
d L_{f} h=\frac{\partial h}{\partial x} f .
$$

In the case under study, the rows of the matrix $\mathrm{O}$ are given by:

$$
\begin{aligned}
& d h=\left[\begin{array}{cc}
\frac{R_{2} R_{3}}{\gamma} & \frac{R_{1} R_{2}}{\gamma}
\end{array}\right]=\left[\begin{array}{ll}
\alpha_{1} & \alpha_{2}
\end{array}\right] \\
& d L_{f} h=\left[\begin{array}{cc}
\frac{\alpha_{3} x_{1}^{2}+\alpha_{4} x_{1}+\alpha_{5} x_{2}+\alpha_{6}}{\left(\alpha_{7} x_{1}+\alpha_{8}\right)^{2}} & \frac{\alpha_{9} x_{1}+\alpha_{10}}{\alpha_{7} x_{1}+\alpha_{8}}
\end{array}\right]
\end{aligned}
$$

Where all $\alpha_{1}$ are defined in eq. (11).

\section{Acknowledgement}

This paper has been funded by the following research projects: 1. RITmare, Ricerca ITaliana per il mare (Italian Research for the sea) CUP:B91J11000740001; 2. TESEO, Tecnologie ad alta Efficienza per la Sostenibilità Energetica ed ambientale On-board (High efficiency technologies for on-board energy and environmental sustainability) CUP: B61C12000850005.

\section{References}

[1] L. Zubieta, R. Bonert. "Characterization of double-layer capacitors for power electronics applications", IEEE Trans. Ind. Appl. 36, 2000.

[2] A. Kitahara, A. Watanabe. "Electrical Phenomena at Interfaces: Fundamentals, Measurements and Applications". New York: Marcel Dekker, 1984.

[3] R. Morrison. "The Chemical Physics of Surfaces". New York: Plenum, 1990.

[4] R. Faranda. "A new parameters identification procedure for simplified Double Layer Capacitor two-branch model”, Elsevier Electric Power Systems Research, Volume 80, Issue 4, April 2010, Pages 363-371.

[5] D. Casadei, G. Grandi, C. Rossi. "A supercapacitor based power conditioning system for power quality improvement and uninterruptible power supply", Proceedings of the IEEE International Symposium on Industrial Electronics (ISIE 2002), July 8-11, 2002, pp. 1247-1252.

[6] S. Buller; E. Karden; D. Kok; R. W. De Doncker. "Modeling the dynamic behavior of supercapacitors using impedance spectroscopy", IEEE Trans. on Industry Applications, Volume: 38, Issue: 6, 2002, pp. $1622-1626$

[7] V. Mugolino, L. Piegari, E. Tironi. "New Full-Frequency-Range Supercapacitor Model With Easy Identification Procedure", IEEE Trans. on Volume: 60 , Issue: 1, 2013, pp. $112-120$.

[8] B.E. Conway. "Electrochemical Supercapacitors: Scientific Fundamentals and Technological Applications". Berlin: Springer. ISBN 0306457369. 1999, retrieved May 2, 2013.

[9] M. S. Halper, J.C. Ellenbogen. "Supercapacitors: A Brief Overview" MITRE Nanosystems Group. 2006, retrieved 2014-01-20. http://ww w.mitre.org/sites/default/files/pdf/06_0667.pdf

[10] A. M. Namisnyk. "A survey of electrochemical supercapacitor technology" . Retrieved 2013-04-02. http://services.eng.uts.edu.au/c empe/subjects_JGZ/eet/AUPEC'03\%20Paper_AN.pdf
[11] Z. Yicheng, L. Haiquan, X. Haitao, W. Lulu. 2008. "Analysis of the time-domain and frequency-domain models of supercapacitors" Vehicle Power and Propulsion Conference, 2008. VPPC '08.

[12] M. Pucci, G. Vitale, G. Cirrincione, M. Cirrincione. "Parameter Identification of a Double-Layer-Capacitor 2-Branch Model by a Least-Squares Method". Industrial Electronics Society, IECON 2013, 39th Annual Conference of the IEEE.

[13] A.S. Weddell, G.V. Merrett, T.J. Kazmierski, B.M. Al-Hashimi. "Accurate supercapacitor modeling for energy-harvesting wireless sensor nodes". IEEE Transactions on Circuits and Systems II: Express Briefs, vol. 58, no. 12, December 2011.

[14] B. L. Walcott , M. J. Corless, S. H. ŻAK. "Comparative study of non-linear state-observation techniques" . International Journal of Control, 1987.

[15] H. K. Khalil. "Nonlinear Systems, Ed.III". Department of Electrical and Computer Engineering, Michigan State University, 2002, 1996 by Prentice Hall, Inc. Upper Saddle River, NJ 07458.

[16] R.S. Borges, A.L.M. Reddy, M-T.F. Rodrigues, H. Gullapalli, K. Balakrishnan, G.G. Silva, P. M. Ajayan. "Supercapacitor operating at 200 degrees celsius", Sci Rep 2013;3:2572-7.arac, http://www.researchgate.net/publication/256377518_Supercapacitor_ operating_at_200_degrees_celsius.

[17] H. Gualous, H. Louahlia, R. Gallay, "Supercapacitor Characterization and Thermal Modelling With Reversible and Irreversible Heat Effect", IEEE Transactions on power electronics, vol 26, no. 11, November 2011. 